\section{Correlation of cumulative corticosteroid treatment with magnetic resonance imaging assessment of avascular femoral head necrosis in patients with multiple sclerosis}

\author{
Nilufer Kale, Jale Agaoglu, 2,3 \\ Osman Tanik ${ }^{2,3}$
}

'Department of Neuro-Ophthalmology, Michigan State University, East Lansing, MI, USA;

'Department of Neurology, Apex Medical and Research Center, Istanbul; ${ }^{3}$ Department of Neurology, Okmeydani Training and Research Hospital, Istanbul, Turkey

\section{Abstract}

Increased risk of osteoporosis, fractures, and avascular necrosis (AVN) has been suggested in multiple sclerosis (MS). Patients with MS are often exposed to corticosteroid treatment (CST) during the disease course and conflicting reports exist regarding complications of CST. Our study aims to investigate the association between cumulative doses of CST and radiographic evaluation of AVN of the femoral head in MS. Twenty-six MS patients (mean age, $38.4 \pm 10 \mathrm{yr}$ ) were enrolled and prospectively evaluated for AVN by magnetic resonance imaging (MRI). The mean disease duration was $11.5 \pm 8.5$ years and mean expanded disability status scale (EDSS) score was $3 \pm 2$. The cumulative dosage of CST varied between $20 \mathrm{~g}$ and $60 \mathrm{~g}$; patients were grouped into two categories: 1) CST between 20-40 g, 17 (65\%) patients; 2) CST $\geq 40$ g; 9 (35\%) patients. The relationship between cumulative CST dosage and MRI diagnosis of AVN was statistically insignificant $(\mathrm{P}>0.9)$. Clarification of the cumulative effect of CST in the development of AVN is of great importance for future long-term steroid treatment strategies.

\section{Introduction}

An increased risk of osteoporosis, fractures, and avascular necrosis (AVN) has been suggested in cases of multiple sclerosis (MS). ${ }^{1-3}$ Although the exact mechanism of AVN is poorly understood, the most common etiological event is reported as trauma and most of the non-traumatic AVN cases are related to longterm corticosteroid treatment (CST). However, the impact of dosage and duration of CST is not apparent. Patients with MS are often exposed to pulse methylprednisolone applications during the disease course and conflicting reports exist regarding complications of $\mathrm{CST}^{4}{ }^{4}$ Our study aims to investigate the association between the cumulative dosage of CST and AVN of the femoral head in MS.

\section{Materials and Methods}

This study was carried out in the setting of a MS center at the Okmeydani Training and Research Hospital, Istanbul. After approval was obtained from the Okmeydani Training and Research Hospital ethical committee, 26 clinically definite MS patients ${ }^{5}$ were identified retrospectively from a larger cohort of patients who were followed in the MS outpatient clinic. Identified patients were evaluated prospectively for AVN. Asymptomatic patients who received at least $20 \mathrm{~g}$ methylprednisolone during the whole course of the disease and with no known AVN were enrolled into the study. The inclusion criteria included patients who did not have an acute attack during the past four weeks or any steroid pulse treatment three months prior to the study. Patients were excluded if comorbidities including thyroid or parathyroid abnormalities, diabetes mellitus, renal failure, hypertension, and autoimmune rheumatic diseases were present. The purpose of the test was explained fully to the patients and informed consent was obtained. Patients were evaluated by an experienced neurologist by routine neurological examination including assessment using the expanded disability status scale (EDSS). ${ }^{6}$ AVN of the femur was assessed bilaterally by magnetic resonance imaging (MRI) by a radiologist blinded to the clinical characteristics. A 1.5-TGE Signo Excite MRI device with $0.4 \mathrm{~mm}$ sections in coronal, transverse, and sagittal planes was utilized. The Ficat Arlet classification was used for radiological evaluation and all the signs of the particular grading system were required to be present for grading. ${ }^{7-9}$ Patients with grades 3 and 4 were accepted as presenting with a radiographic diagnosis of AVN (RAVN) (see Appendix). Biochemical bone markers including serum calcium, magnesium, and phosphorus, alkaline phosphates (ALP), serum parathyroid hormone (PTH) level, and 25hydroxyvitamin D (25(OH)D) were also assessed.

\section{Statistical Analysis}

Descriptive statistics were applied to the demographic features of the cohort. The
Correspondence: Nilufer Kale, 1626 Spartan Village Apt J, 48823 East Lansing, MI, USA.

E-mail: kalenilufer@yahoo.com

Key words: multiple sclerosis, corticosteroid treatment, avascular femoral head necrosis.

Contributions: NK, JA: participation in the study; NK: manuscript preparation; NK, OT: manuscript edition.

Conflict of interest: the authors report no conflicts of interest.

Received for publication: 22 January 2010.

Revision received: 13 August 2010.

Accepted for publication: 20 August 2010.

This work is licensed under a Creative Commons Attribution 3.0 License (by-nc 3.0).

(C) Copyright N. Kale et al., 2010

Licensee PAGEPress, Italy

Neurology International 2010; 2:e17

doi:10.4081/ni.2010.e17

Wilcoxon rank test was applied for continuous variables and the Kruskal-Wallis rank test was used for ordinal and continuous variables. The Chi-square test (normally distributed parameters) and Mann-Whitney U test (not normally distributed parameters) were used to compare the groups. A $\mathrm{P}<0.05$ was considered statistically significant.

\section{Results}

We identified 26 clinically definite MS patients ( 15 females, $58 \%$ ), with a mean age of $38.4 \pm 10$ years. ${ }^{5}$ The mean disease duration was $11.5 \pm 8.5$ years and the mean EDSS score for the patient group was $3 \pm 2$ (Table 1). Biochemical determinants were found to be within the normal range (Table 2). There was no relationship between bone biochemical markers and RAVN. ${ }^{10}$ The cumulative dosage of CST varied between $20 \mathrm{~g}$ and $60 \mathrm{~g}$, and the patients were grouped into two categories as follows: 1) CST of 20-40 g, 17 (65\%) patients; 2) CST $\geq 40$ g, 9 (35\%) patients. The total number of patients with RAVN was 6 (23\%). The distribution of the patients with RAVN according to CST dosage was as follows: 1) CST of 20-40 g, 4 patients (15\%) had RAVN; 2) CST $\geq 40$ g, 2 patients (8\%) presented with RAVN. The relationship between cumulative CST dosage and RAVN was statistically insignificant $(\mathrm{P}>0.9)$. However, a statistically significant association was observed between cumulative CST dosage, age, EDSS, and disease duration $(\mathrm{P}<0.0001)$ (Table 3$)$. 


\section{Discussion and Conclusions}

We aimed to raise the question of the effects of the cumulative CST on the development of RAVN. Recent studies implicated high-dose CST for immunosuppression after organ transplantation, as well as for rheumatological and autoimmune diseases, as a risk factor for development of atraumatic AVN. ${ }^{11-13}$ The most common reason for steroid application is generally rheumatic diseases like systemic lupus erythematosus or rheumatoid arthritis. The discussion over the exact nature of AVN in these diseases, a vasculitic process or CST, is still an unresolved issue., ${ }^{4,14}$ In one study, the frequency of AVN between the treatment group and the controls was investigated and found to be statistically significant, which supported the claim that high doses of steroids given in a short time course would constitute an independent risk for AVN. ${ }^{4}$ Another study evaluated the frequency of AVN among kidney transplant recipients and showed that the cumulative steroid dosage was higher among the AVN group than the control group. The study suggested that reduction or early withdrawal of steroids was the only efficient preventive treatment for AVN. ${ }^{13}$ In our study, we failed to demonstrate a significant relationship between RAVN and cumulative steroid dosage. However, our diagnosis was based on radiographic findings and clinical presentation was not evaluated. In addition, it would be useful to investigate a correlation between the dosage of CST used and the grade of AVN. For our study purposes, only the presence of RAVN (patients with AVN grades 3 and 4) was documented and a correlation between grading of RAVN and CST dosage was not assessed further in the study group. Small sample size and lack of a control group are also limitations of our report and studies with a longer term follow-up in a large cohort are warranted. However, our study aimed to investigate a very important question about the dose-related effects of CST and, to date, this issue has not been clarified. Clarification of the cumulative effect of CST in the development of AVN is of great importance for future long-term steroid treatments in MS as well as for routine clinical practice where repeated CST is administered.

\section{Appendix}

- Grade 0: no clinical and X-ray findings, double-line sign on MRI.

- Grade 1: joint is normal. The femoral head is spherical with slight osteoporosis on $\mathrm{X}$ ray and a single line on $\mathrm{T} 1$ and double line on T2, which demarcates necrotic bone.

- Grade 2: double-line sign, reactive bone

Table 1. Demographic and clinical characteristics.

\begin{tabular}{ll}
\hline Gender (F:M) & $2: 1$ \\
Age (yr) & $38.4 \pm 10$ \\
\hline DD (yr) & $11.5 \pm 8.5$ \\
Dx (\%) & $17(63)$ \\
RRMS & $9(27)$ \\
SPMS & $3 \pm 2$ \\
\hline EDSS & $17(65 \%)$ \\
\# Patients according to cumulative CST (\%) & $9(35 \%)$ \\
$20-40 \mathrm{~g}$ & \\
$>40 \mathrm{~g}$ & $4(15 \%)$ \\
\hline Distribution of AVN (\%) & $2(8 \%)$ \\
$20-40 \mathrm{~g}$ & \\
$>40 \mathrm{~g}$ &
\end{tabular}

DD, disease duration; RRMS, relapsing, remitting MS; SPMS, secondary progressive MS; EDSS, expanded disability status scale; CST, corticosteroid; AVN, avascular bone necrosis.

Table 2. Biochemical bone markers in the patient group.

\begin{tabular}{lccc} 
& Normal range & Results & Significance \\
Calcium $(\mathrm{mg} / \mathrm{dL})$ & $9-10.6$ & $9.7 \pm 0.5$ & $\mathrm{P}>0.25$ \\
Phosphorus $(\mathrm{mg} / \mathrm{dL})$ & $2.5-45$ & $4.6 \pm 2.2$ & $\mathrm{P}>0.5$ \\
\hline Magnesium $(\mathrm{mg} / \mathrm{dL})$ & $1.2-2.5$ & $1.6 \pm 0.7$ & $\mathrm{P}>0.6$ \\
PTH $(\mathrm{ng} / \mathrm{mL})$ & $0.15-0.6$ & $0.4 \pm 0.1$ & $\mathrm{P}>0.95$ \\
\hline $25(\mathrm{OH}) \mathrm{D}(\mathrm{ng} / \mathrm{mL})$ & $11-45$ & $37.4 \pm 4.3$ & $\mathrm{P}>0.3$ \\
ALP $(\mathrm{U} / \mathrm{L})$ & $43-122$ & $65.7 \pm 14.4$ & $\mathrm{P}>0.5$
\end{tabular}

Values are expressed as the mean with standard deviation and P-values. PTH, parathyroid hormone; $25(\mathrm{OH})$ D, 25-hydroxvitamin D; ALP, alkaline phosphates.

Table 3. Distribution of demographics and clinical data according to cumulative corticosteroid treatment dosage.

\begin{tabular}{lcc} 
& Cumulative CST dose $20-40 \mathrm{~g}$ & Cumulative CST dose $\geq \mathbf{4 0} \mathrm{g}$ \\
Age & $32.3 \pm 5.5$ & $50.0 \pm 4.4$ \\
DD & $6.3 \pm 3.2$ & $21.5 \pm 6.0$ \\
\hline EDSS & $1.9 \pm 0.8$ & $5.9 \pm 0.95$
\end{tabular}

Values are expressed as the mean with standard deviation. CST, corticosteroid treatment; EDSS, expanded disability status scale; $\mathrm{DD}$, disease duration.

demarcates the area of infarct. The joint is normal and the femoral head is still spherical.

- Grade 3: loss of the spherical shape of the femoral head, fracture of subchondral bone, and crescent sign on X-ray.

- Grade 4: further collapse of the femoral head, articular cartilage destruction, and joint space narrowing.

\section{References}

1. Zorzon M, Zivadinov R, Locatelli L, et al. Long-term effects of intravenous high dose methylprednisolone pulses on bone mineral density in patients with multiple sclerosis. Eur J Neurol 2005;12:550-6.

2. Stepan JJ, Havrdova E, Tyblova M, et al. Markers of bone remodeling predict rate of bone loss in multiple sclerosis patients treated with low dose glucocorticoids. Clin Chim Acta 2004;348:147-54.

3. Stenager E, Jensen K. Fractures in multiple sclerosis. Acta Neurol Belg 1991;91: 296-302.

4. Ce P, Gedizlioglu M, Gelal F, et al. Avascular necrosis of the bones: an overlooked complication of pulse steroid treatment of multiple sclerosis. Eur J Neurol 2006;13:85761 .

5. Poser CM, Paty DW, Scheinberg LC, et al. New diagnostic criteria for multiple sclerosis: guidelines for research protocols. Ann Neurol 1983;13:227-31.

6. Kurtzke JF. Rating neurologic impairment in multiple sclerosis: an Expanded Disability Status Scale (EDSS). Neurology 1983;33:1444-52.

7. Ficat RP. Idiopathic bone necrosis of the femoral head: early diagnosis and treat- 
ment. J Bone Joint Surg Br 1985;67:3-9.

8. Nakanishi K, Tanaka H, Sugano N, et al. MR-based three-dimensional presentation of cartilage thickness in the femoral head. Eur Radiol 2001;11:2178-83.

9. Thickman D, Axel L, Kressel HY, et al. Magnetic resonance imaging of avascular necrosis of the femoral head. Skeletal Radiol 1986;15:133-40.

10. Tuzun S, Altintas A, Karacan I, et al. Bone status in multiple sclerosis: beyond corticosteroids. Multiple Sclerosis 2003;9:6004.

11. Bekler H, Uygur AM, Gökçe A, et al. The effect of steroid use on the pathogenesis of avascular necrosis of the femoral head: an animal model. Acta Orthop Traumatol Turc 2007;41:58-63.

12. Karapinar L, Gurkan A, Kacar S, et al. Posttransplant femoral head avascular necro- sis: 4 selective investigations with MRI. Ann Transplant 2007;12:27-31.

13. Hedri H, Cherif M, Zouaghi K, et al Avascular osteonecrosis after renal transplantation. Transplant Proc 2007;39:1036-8.

14. Mok MY, Farewell VT, Isenberg DA. Risk factors for avascular necrosis of bone in patients with systemic lupus erythematosus: Is there a role for antiphospholipid antibodies? Ann Rheum Dis 2000;59:462-7. 